\title{
Transient Electromagnetic Field Characteristics of A New Source-Conical Source
}

\author{
Haiyan Yang, Fengping Li \\ State Key Laboratory Breeding Base of Nuclear Resources and Environment, \\ East China University of Technology, Nan Chang, China \\ genious_yang@126.com,
}

\begin{abstract}
The mutual inductance coupling of the traditional multi-turn coils is relatively large, which makes the shallow "blind area" of the detection increase. Therefore, a conical field source device was proposed, and the calculation formula of the radius of each turn coil in the device was given. First of all, the theoretical analytic expression was used to calculate and analyze the characteristic of primary field and secondary field of the conical field source in homogeneous medium, and then the comparison of the mutual inductance of the conical field source device and the multi-turn coils was carried out. After that, the fast Hankel transform and improved cosine transform numerical filtering algorithm were applied to calculate the electromagnetic response of horizontal layered earth under excitation of the step current. Finally, the induced electromotive force was converted into a vertical magnetic field, and then the apparent resistivity of the layered medium was obtained. The study found that the mutual inductance of multi-turn coils is about 9 times of the conical field source's, which have the same equivalent magnetic moment of 926.1Am2. The characteristic of primary and secondary field of the conical field source is the same as that of the multi-turn coils; The magnetic field intensity of conical field source and multi-turn coils differs by two orders of magnitude under the three layers geoelectric model of $\mathrm{H}$-type, but both of similar characteristic, and apparent resistivity that is consistent with the model can be available.
\end{abstract}

Keywords-transient electromagnetic method; cone-shaped source; transient process; Apparent resistivity; Mutual inductance

\section{INTRODUCTION}

The traditional small device of transient electromagnetic mainly consists of a common center point device and a dipole device, in order to enhance the initial electromagnetic energy, Multi-turn winding is usually used to increase the magnetic moment. At this time, the strong mutual coupling at the interior of the coil and between the coils makes turn-off effect enhanced and shallow "blind area" increase (20-30m). For this reason, the traditional transmitting device was changed into a conical shape, and the radius of each turn coil was increased by a small increase, and a certain distance is separated, thereby reducing the mutual inductance between the coils and reducing the range of the "blind area".

\section{CONICAL FIELD SOURCE}

The approximate representation of the conical field source is available by fig.1a), it consists of a $\mathrm{n}$ radius of the charged ring between $\mathrm{r} 1$ and $\mathrm{r} 2$, the current intensity of the ring is I, the distance between the top ring and the bottom ring is D. When the turn number $\mathrm{n}$, distance $\mathrm{D}$, radius $\mathrm{R} 1$ and $\mathrm{R} 2$ are determined, the radius of each turn circle ring and the distance between the center of each circle turns can be determined.

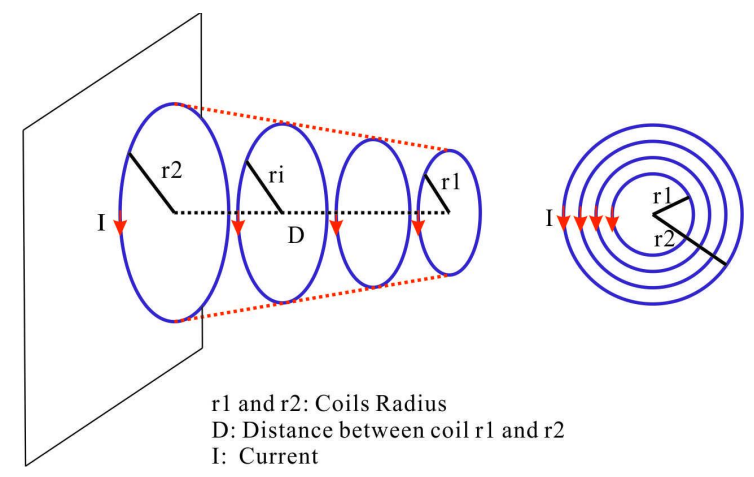

Fig.1 Theoretical model of field source device

It is easy to obtain the distance d of two adjacent coils between the center and the radius $r_{i}$ of the $i$-th coil as follows

$$
d=\frac{D}{n-1} \text { and } r_{i}=r_{1}+\frac{i\left(r_{2}-r_{1}\right)}{n-1}
$$

It can be found from the above equation that the radius $r_{i}$ of the $\mathrm{i}$-th coil is constrained only by the top and bottom radius $\mathrm{r} 1, \mathrm{r} 2$ and turns $\mathrm{n}$. In order to correspond with the size of the experimental device, the height, the top coil radius and the bottom coil radius of the conical device model were separate $1 \mathrm{~m}, 0.5 \mathrm{~m}, 1 \mathrm{~m} .101$ turns coils were wound so that the coil radius is calculated without recurring decimal, and the calculation errors arising therefrom can be avoided. $5 \mathrm{~A}$ current is passed through the coil, the equivalent magnetic moment $\mathrm{M}_{0}$ of the model is $926.1 \mathrm{Am}^{2}$.

\section{Field SOURCE CHARACTERISTICS}

When a current I passing through a single turn circular loop, the vertical component of the magnetic field at any point in space as follows ${ }^{[1]}$

$$
B_{z}=\frac{\mu I r_{0}^{2}}{4} \cdot \frac{2 r_{0}^{2}-\left(x^{2}+y^{2}-2 z^{2}\right)}{\left(r^{2}+r_{0}^{2}\right)^{5 / 2}}
$$

Where $\mu$ is the magnetic permeability of the medium, which may generally be taken as vacuum permeability $\mu_{0}$ in a non- 
magnetic medium. $r_{0}$ is the radius of the circle loop, $r$ is the distance from any point in space to the center of circular loop. If the center of circular loop is located in the origin of coordinates, then $r=\sqrt{x^{2}+y^{2}+z^{2}}$.

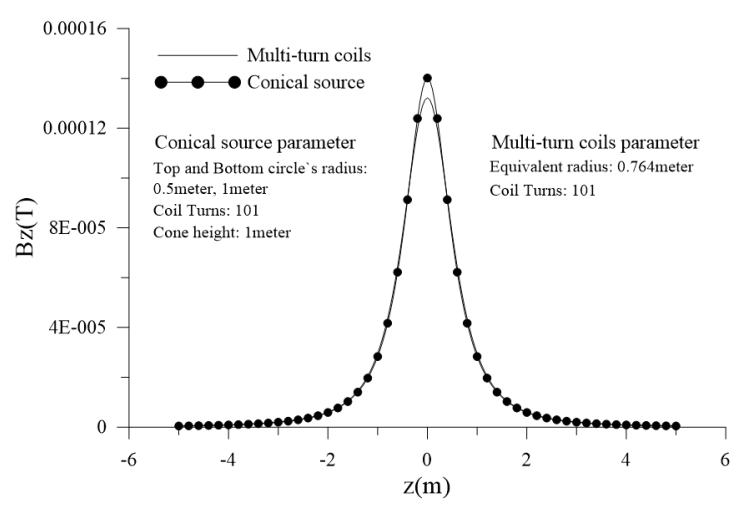

Fig.2 Comparison of Bz component of conical and multi-turn coils

The height, the top coil radius and the bottom coil radius of the conical device model were separate $1 \mathrm{~m}, 0.5 \mathrm{~m}, 1 \mathrm{~m}$, total 101 turns coils; Thereby the equivalent radius of multi-turn coils which have the same moment and the same number of turns is 0.764 meters. A magnetic induction calculated with the parameters of the two device is shown in Fig.2, the abscissa of the figure is the axial direction (That is the z-axis) of the two field source. Magnetic field at the center of the field source is the strongest, and the magnetic field is gradually weakened when the spot is far away from the center of the field source. The magnetic field intensity of the conical field source is higher than that of the multi-turn coils at the center of the field source, due to the center point of each turn coil is not coincident. Overall, the primary field produced by the two devices of the same magnetic moment has achieved a better fit in the axial direction.

The theoretical secondary field of Transient Electromagnetic was calculated with the same device parameters as Fig. ${ }^{[2]}$, the corresponding expression (3) of the center of the loop in uniform half space was used for the analytical expression of the time derivative of the magnetic field. It can be found that the theoretical secondary field of conical field source and multi-turn coils is the same (Fig.4) in the condition of equal magnetic moment. Therefore, the conical field source theoretically can achieve the transmission effect of multi-turn coils source.

$$
\frac{\partial B_{z}(t)}{\partial t}=\frac{I_{0} \rho}{r_{0}^{3}}\left[3 \Phi(u)-\frac{2}{\sqrt{\pi}} e^{-u^{2}} u\left(3+2 u^{2}\right)\right]
$$

Where, $I_{0}$ is the intensity of the supply current, $t$ is the observation time, $r_{0}$ is the radius of the transmitter coil. Simultaneously,

$$
\begin{gathered}
\Phi(u)=\frac{2}{\sqrt{\pi}} \int_{0}^{u} e^{-x^{2}} d x, \\
u=\frac{\pi \sqrt{2} r_{0}}{\tau}, \tau=2 \pi \sqrt{2 \rho t / \mu}
\end{gathered}
$$

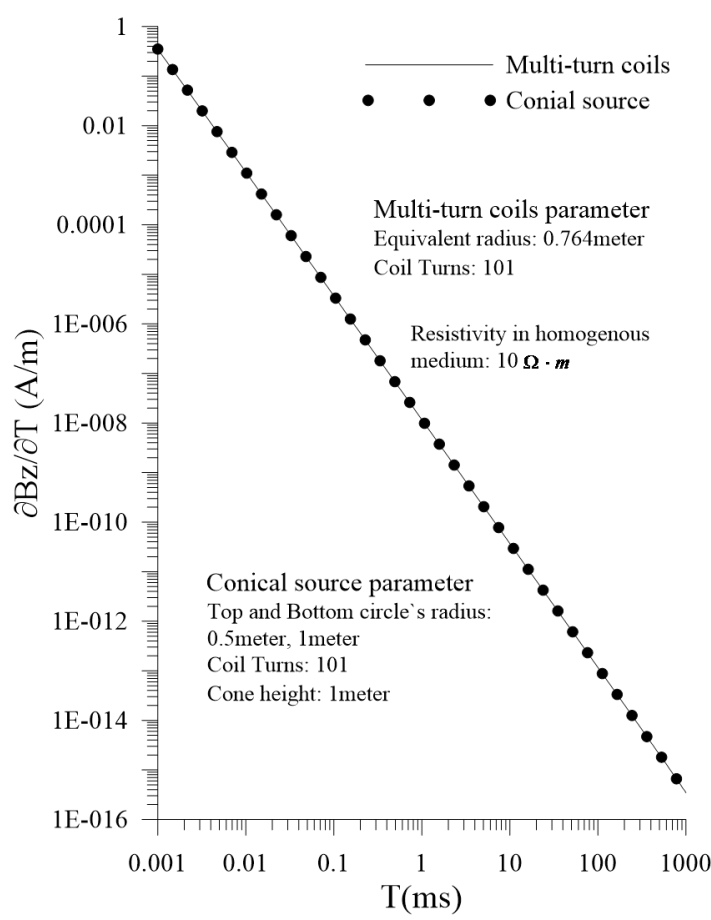

Fig. 3 Curves of Secondary magnetic field
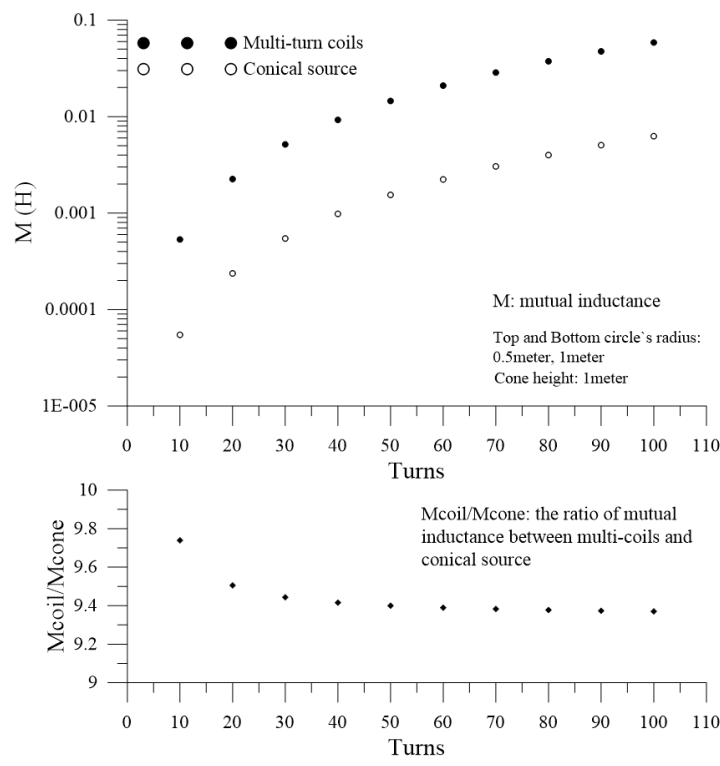

Fig.4 Coefficients of mutual inductance of conical field source and multi-turn coils 


\section{Mutual InduCtance Characteristics}

The height, the top coil radius and the bottom coil radius of the conical device model were separate $1 \mathrm{~m}, 0.5 \mathrm{~m}, 1 \mathrm{~m}$, Turns increased from 10 to 100 , according to the total magnetic moment is equal, the equivalent radius of the multiturn coils of corresponding turns was calculated. Fig.4 shows that the mutual inductance of the two devices gradually increased with the increase of turns, but the ratio of mutual inductance of multi-turn coils and conical field source is greater than 9.3, that is the mutual inductance of multi-turn coils is always higher than that of the conical field source more than 9.3 times. Thus, the change of the shape of field source greatly weakens the mutual inductance between the coils.

\section{FORWARD}

The Bessel function was calculated with the Fast Hankel transform of D.Guptasarma and B.Singh, and using the improved cosine transform numerical filtering algorithm ${ }^{[4]}$ to calculate the cosine integral, thus the response of horizontal layered earth electromagnetic under excitation of the step current was calculated. In the computation of apparent resistivity, first the induction electromotive force was converted into the vertical magnetic field ${ }^{[5]}$ by formula (4), then the calculation of late-time apparent resistivity.

$$
H_{z}(t)=\frac{1}{S n \mu_{0}} \int_{t}^{b} V_{z}(t) d t
$$

Where, $S$ is the area of the receiving coil, $n$ is the number of turns.

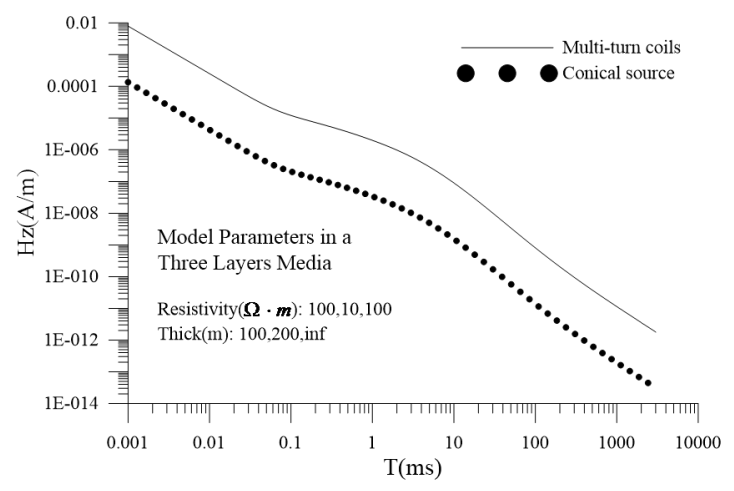

Fig.5 Curves of magnetic field intensity of $\mathrm{H}$ type model

The three layers geoelectric model of H-type was established to carry out the forward of multi-turn coils and conical device, equivalent magnetic moment of the two devices is $926.1 \mathrm{Am}^{2}$, and the equivalent radius of multi-turn coils was calculated to be 0.764 meters. The results of forward model show that the magnetic field intensity of the two devices differs by two orders of magnitude, but both of similar characteristics. After being converted into apparent resistivity, its characteristics coincide quite well with the model resistivity, thereby obtaining a new method for calculating the apparent resistivity of conical field source.

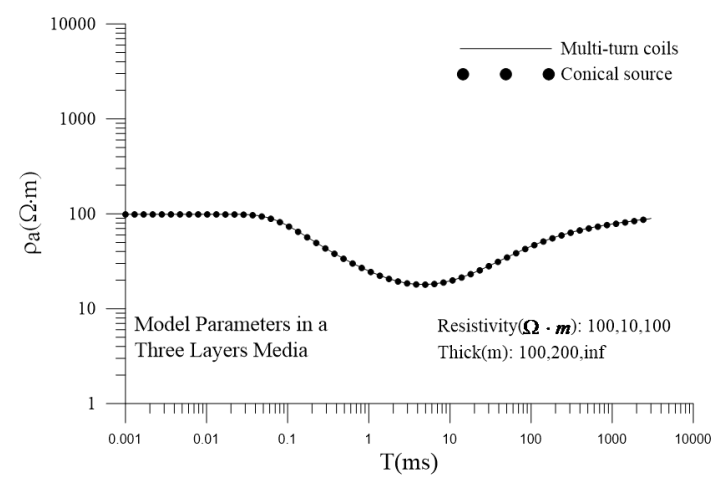

Fig. 6 Curves of apparent resistivity of $\mathrm{H}$ type model

\section{CONCLUSION}

A new type of field source was proposed, which was based on the weakening of the mutual inductance between the coils and the realization of the same detection effect. After theoretical study of the field source, we found that: First of all, for the conical field source which the height, the top coil radius and the bottom coil radius were separate $1 \mathrm{~m}, 0.5 \mathrm{~m}, 1 \mathrm{~m}$, its mutual inductance is much less than that of the multi-turn coils with the same magnetic moment, which is about $1 / 9$ of a multi-turn coils. What's more, the characteristics of primary and secondary field of the conical field source are the same as that of the multi-turn coils. Last but not least, the magnetic field intensity of conical field source and multi-turn coils differs by two orders of magnitude for the three layers geoelectric model of H-type, but both of similar characteristics, and apparent resistivity that is consistent with the model can be available.

\section{ACKNOWLEDGMENT}

The authors would like to express their profound thanks to Jianhua YUE at China University of mining and technology and Guoqiang XUE at the Institute of Geology and Geophysics, Chinese Academy of Sciences. The project was supported by the National Natural Science Foundation of China (Grant No.41564001) and the Natural Science Foundation of Jiangxi Province (Grant No.20151BAB203045).

\section{REFERENCES}

[1] Haiyan Yang. Study on numerical simulation and distribution regularity of transient electromagnetic field with Ming-used multi small loop [Ph.D. thesis].Jiangsu Xuzhou: China University of Mining Technology, 2009.)

[2] Kaufman A A, Eaton P A. The Theory of Inductive Prospecting. Elsevier, 2001.

[3] Guptasarma, B.Singh. New digital linear filters for Hankel J0 and J1 transforms [J]. Geophysical Prospecting [J],45: 745-762.

[4] WANG Huajun.2004. Digital filtering algorithm of sine and cosine transform [J]. Engineering Geophysics, 51(6): 1936 1942.

[5] CHEN Mingsheng, TIAN Xiaobo. Study on the transient electromagnetic (TEM) sounding with electric dipole.V. The measured induced voltage transformed to the vertical magnetic field [J]. Goal Geology and Exploration, 1999, 27(5):63-65. 